\title{
JEFF-3.3 covariance application to ICSBEP using SANDY and NDAST
}

\author{
Luca Fiorito ${ }^{1, *}$, James Dyrda ${ }^{1}$, and Michael Fleming ${ }^{1}$ \\ ${ }^{1}$ OECD / Nuclear Energy Agency, 45 Quai Alphonse le Gallo, Boulogne-Billancourt, 92100, France
}

\begin{abstract}
Providing reliable estimates of the nuclear data contribution to the uncertainty of well-known integral benchmarks is fundamental to the validation and verification process for a nuclear data library. The Nuclear Energy Agency has produced and maintains the NDaST sensitivity tool, which integrates the DICE sensitivities and nuclear data covariances. This system has been used to rigorously and efficiently provide direct feedback to evaluators and streamline validation. For its future evolution and to identify high-priority development areas, NDaST is continuously compared against state-of-the-art codes that use different uncertainty propagation methodologies.

In this work, NDaST was compared to the nuclear data sampling code SANDY for several ICSBEP criticality benchmarks using the JEFF-3.3 evaluated data. Despite excellent overall agreement for cross sections and fission neutron multiplcities, discrepancies due to processed covariance descriptions for angular distributions and prompt fission neutron spectra have identified areas where coordinated development of nuclear data covariance descriptions should be prioritised.
\end{abstract}

\section{Introduction}

The International Criticality Safety Benchmark Evaluation Project (ICSBEP) [1] provides a compilation of critical and subcritical benchmark experiment data that are maintained and continuously updated by the OECD/Nuclear Energy Agency (NEA) Working Party on Nuclear Criticality Safety (WPNCS). Nuclear data evaluation projects such as JEFF and ENDF/B regularly use these benchmarks to validate their nuclear data libraries. The validation process includes assessing the impact of nuclear data on the uncertainty of the benchmark integral responses, often with a focus on $\mathrm{k}_{\mathrm{eff}}$. Nuclear data evaluators have made significant efforts to include covariances for several data types into the official general-purpose nuclear data libraries. For example, the latest released library of the Joint Evaluated Fission and Fusion (JEFF) project, JEFF-3.3, contains 562 evaluations including:

- 50 covariance files for fission neutron multiplicities;

- 352 covariance files for resonance parameters;

- 442 covariance files for cross sections;

- 359 covariance files for angular distributions;

\footnotetext{
*e-mail: luca.fiorito@oecd-nea.org
} 
- 36 covariance files for energy distributions; and

- 286 covariance files for activation cross sections.

In parallel to the development of more complete nuclear data evaluations and covariances, computer codes have been developed to process and propagate these covariances to be able to quantify the uncertainty on various neutronics parameters, including $\mathrm{k}_{\mathrm{eff}}$. Two uncertainty propagation approaches have been widely implemented in these codes: linear perturbation theory and Monte Carlo uncertainty propagation. The first method requires calculating the sensitivity of simulated quantities to the nuclear data, a feature that has been added to several neutron transport codes such as MCNP [2], SCALE (TSUNAMI) [3], ERANOS [4] and SERPENT [5]. The second refers to computational approaches that rely on the repeated random sampling of chosen parameters to obtain statistical outcomes of selected responses. Several codes have been developed to sample the nuclear data evaluations, including SANDY [6], SAMPLER[7], XSUSA [8], NUDUNA [9] and NUSS [10]. Semi-empirical model codes such as TALYS [11] are also used to generate sampled nuclear data evaluations based on the direct sampling of parameters used within physics models. This has the added benefit of allowing complete freedom in the distributions of quantities in nuclear data that are often assumed to have Gaussian distributions.

Today, the NEA Nuclear Data Sensitivity Tool (NDaST) is one of the most efficient codes to propagate nuclear data uncertainties into ICSBEP benchmarks. In this work, the performances of NDaST and SANDY were compared on a suite of ICSBEP cases to cross-validate their methods and identify any potential shortcomings. The study focused on newly implemented features using sensitivities to angular distributions and the propagation of these uncertainties.

\section{Uncertainty propagation tools}

For this work the JEFF-3.3 data library was processed into BOXER format with NJOY. The BOXER multigroup covariance files were produced on the SCALE 238-energy-group structure and were included in the JANIS database.

All available cross section covariances, P1 elastic scattering ${ }^{1}$, total fission multiplicities and prompt fission neutron spectra (PFNS) covariances were utilised. Although the evaluations for PFNS contain covariance data for different incident-neutron energies, only those for $1 \mathrm{MeV}$ were considered due to limitations in the current NJOY-2016.

Perturbed files were generated with SANDY for all evaluations in the JEFF-3.3 library. Each of these was processed with NJOY into ACE format to be used in MCNP calculations.

\subsection{NDaST}

NDaST is the nuclear data sensitivity tool developed and maintained by the OECD/Nuclear Energy Agency [12]. Amongst is well-known features, NDaST can propagate nuclear data uncertainties and covariances to model responses, such as the $\mathrm{k}_{\mathrm{eff}}$, for most of benchmark models recommended by the ICSBEP. To carry out this task, NDaST interfaces with the JANIS [13] and DICE [14] databases to retrieve nuclear data covariance matrices $\left(C_{N D}\right)$ and $\mathrm{k}_{\text {eff }}$ sensitivity profiles $(S)$, respectively. Uncertainties are propagated using the so-called sandwich formula,

$$
C_{k e f f}=S^{t} C_{N D} S
$$

\footnotetext{
${ }^{1} \mathrm{P} 1$ is the first Legendre polynomial coefficient for the angular distribution of elastic scattering. In the rest of the paper we will use the nomenclature $\mathrm{P} n$ to refer to the coefficient of the Legendre polynomial of order $n$.
} 
The sensitivity profiles stored in DICE were calculated on a 238-multigroup structure with MCNP6 and/or TSUNAMI and cover most of the reaction cross section channels. Sensitivities are also often available for total fission neutron multiplicities, PFNS and P1 elastic scattering.

\subsection{SANDY}

SANDY is a nuclear data sampling tool that, for a given evaluated nuclear data and covariance file formatted according to the standard ENDF-6 rules [15], can produce perturbed copies of the file that statistically reflect the covariance information. The perturbed files are generated by applying random perturbation coefficients sampled from the multivariate Gaussian distribution defined by the evaluated data. SANDY can perturb any type of nuclear data for which covariances are provided, including cross sections, and fission neutron multiplicities, as well as emitted energy and/or angular distributions. SANDY enforces consistency with the conservation rules by renormalizing the probability distribution functions and recalculating derived cross sections ${ }^{2}$.

The perturbed files are suitable for Monte Carlo uncertainty propagation calculations, where a given model is solved repetitively, each time adopting a different file. From the statistical analysis of the resulting output predictions one can calculate the distributions of any calculated quantity, including their mean, variance and any other moments.

\section{Uncertainty propagation with ICSBEP benchmarks}

The two codes and methodologies were cross-validated and compared against a selection of ICSBEP cases. The Jezebel benchmark was selected as a simple but realistic model for comparison, as well as the full set of $19 \mathrm{PMF}^{3}$ cases within the so-called Mosteller suite. The Mosteller suite is a well-known suite of MCNP input files for 119 ICSBEP benchmark models that cover several applications including different fuel types, neutron spectra and reflective materials.

\subsection{Jezebel}

For this test case, only the uncertainties on ${ }^{239} \mathrm{Pu}$ data were propagated, which include covariances for cross sections, fission neutron multiplicities (nubar) and PFNS, but angular distribution covariances were excluded. Table 1 reports the $\mathrm{k}_{\text {eff }}$ uncertainty values calculated with SANDY and NDaST. SANDY was used to generate 1000 perturbed files, which produced a distribution of $\mathrm{k}_{\mathrm{eff}}$ values from which the standard deviation was calculated. A variance of variance estimator was calculated to determine the confidence of this uncertainty value.

An excellent agreement was observed between NDaST and SANDY for cross sections and nubar. The difference found for PFNS generates from the way the PFNS covariances were processed by NJOY into BOXER-formatted files. SANDY perturbed files can be correctly used to propagate the PFNS covariances using the fission spectrum of the system as weighing function without the intermediate data format restrictions imposed by the BOXER format. SANDY reproduces NDaST results by sampling from the PFNS covariance matrix for incident-neutron energy of $1 \mathrm{MeV}$ and extending its validity range to the entire energy domain.

\footnotetext{
${ }^{2}$ Derived cross sections are those that can be reconstituted from other existing reactions using standard summation rules

${ }^{3}$ PU-MET-FAST follows the DICE nomenclature to identify the ICSBEP cases by fissile material (PU=plutonium), fuel physical form (MET=metallic) and neutron spectrum (FAST=fast). PU-MET-FAST is also often shortened as PMF.
} 
Table 1: $\mathrm{k}_{\mathrm{eff}}$ uncertainty prediction for Jezebel using SANDY and NDaST. Uncertainties calculated with SANDY are given together with an estimate of the variance of variance.

\begin{tabular}{lcc}
\hline data type & $\begin{array}{c}\text { NDaST } \\
\text { uncertainty }(\mathrm{pcm})\end{array}$ \\
\hline${ }^{\text {All }}{ }^{239} \mathrm{Pu}$ nuclear data & 604 & $562 \pm 13$ \\
${ }^{239} \mathrm{Pu}$ cross sections & 221 & $221 \pm 5$ \\
${ }^{239} \mathrm{Pu}$ fission neutron multiplicities & 414 & $418 \pm 9$ \\
${ }^{239} \mathrm{Pu}$ prompt fission neutron spectra & 381 & $269 \pm 6$ \\
\hline
\end{tabular}

\subsection{PU-MET-FAST}

Similarly to what was done for Jezebel, NDaST and SANDY were used to quantify the nuclear data contributions to the $\mathrm{k}_{\text {eff }}$ uncertainty for 19 PMF benchmarks. For all the isotopes reported in the selected benchmarks we generated 300 perturbed files with SANDY, which were then used in parallel MCNP calculations. All available covariance matrices and sensitivity profiles for the 19 benchmarks were employed for the NDaST calculation.

Figure 1 displays an overall agreement between the two codes for most cases, although some results were still affected by the BOXER format of the PFNS covariances used by NDaST. The $k_{\text {eff }}$ uncertainties for 16 of the 19 benchmarks range from $500 \mathrm{pcm}$ up to 1000 pcm, while three benchmarks (PMF6, PMF10 and PMF20) exhibited relatively large uncertainties that exceeded $1500 \mathrm{pcm}$ in both SANDY and NDaST calculations.

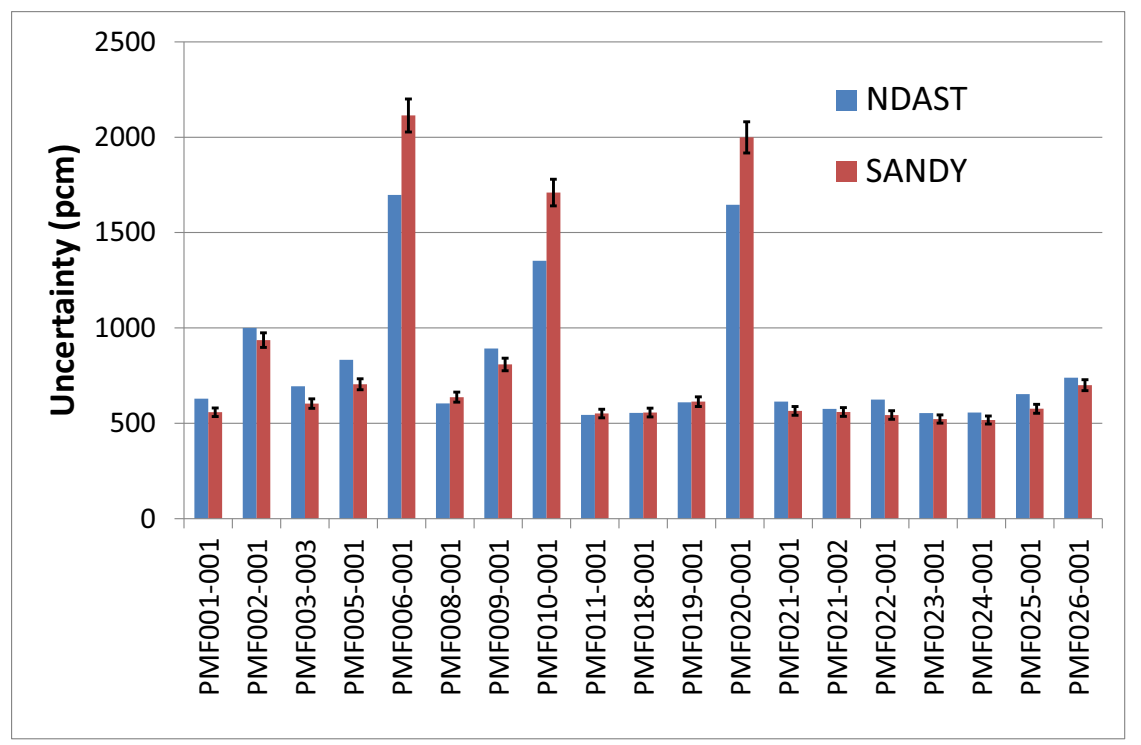

Figure 1: $\mathrm{k}_{\mathrm{eff}}$ uncertainties for ICSBEP PU-MET-FAST benchmarks calculated with NDaST and SANDY. 
The inter-case $\mathrm{k}_{\mathrm{eff}}$ correlations - also known in the nuclear industry as $C_{k}$ values — originate from the nuclear data covariances and were computed using the SANDY simulations, as shown in Figure 2. A strong correlation $>99 \%$ was identified between the same three benchmarks (PMF6, PMF10 and PMF20) with significantly higher nuclear data uncertainty. All three of these benchmarks inlclude a heavy ${ }^{238} \mathrm{U}$ reflector. It is common for systems with important reflectors to have a large $\mathrm{k}_{\mathrm{eff}}$ sensitivity to the neutron leakage term, and consequently to the angular distribution of the neutrons scattered out of the reflected material. In the following section we analyzed this contribution and its impact to the total $\mathrm{k}_{\mathrm{eff}}$ uncertainty for the PMF6 case.

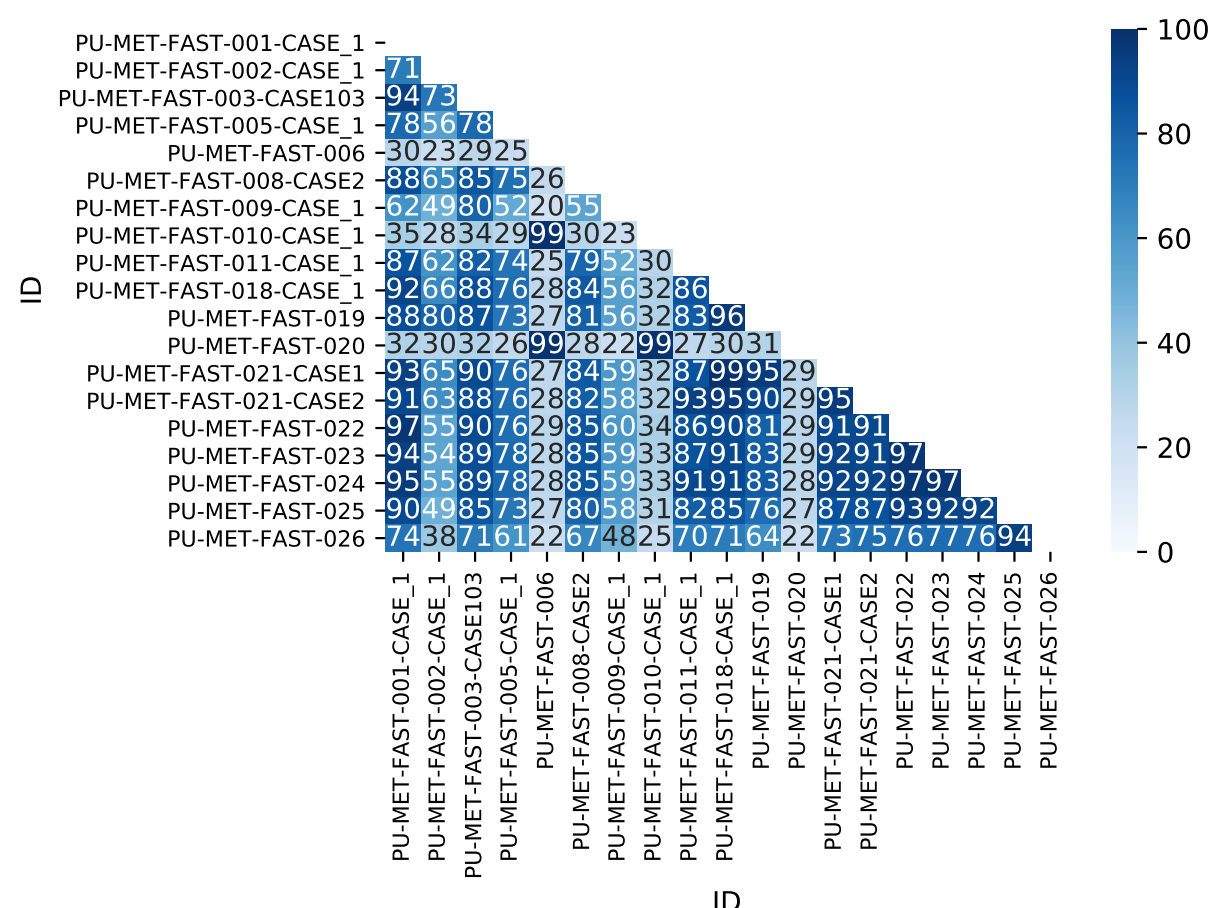

Figure 2: $\mathrm{k}_{\text {eff }}$ correlation matrix for ICSBEP PU-MET-FAST benchmarks generated by nuclear data.

\subsection{PU-MET-FAST-006}

The PMF6 benchmark model is a delta-phase plutonium-alloy sphere with a mass of $6.06 \mathrm{~kg}$ reflected by about $19 \mathrm{~cm}$ of natural uranium. The contribution to the PMF6 $\mathrm{k}_{\mathrm{eff}}$ uncertainty of the angular distributions of neutrons scattered in ${ }^{238} \mathrm{U}$ was assessed with NDaST and SANDY. For the latter, we relied upon 1000 perturbed files that were processed into ACE format and used in MCNP simulations. The covariance matrix taken from JEFF-3.3 includes covariances for the Legendre polynomial coefficients (including cross-coefficient correlations) up to the $6^{\text {th }}$ order. DICE currently contains sensitivity profiles for the first Legendre polynomial coefficient, i.e. P1. As a consequence, NDaST does not propagate the available covariances from P2 to P6. 
The uncertainties calculated with SANDY and NDaST were 2056 \pm 46 and $1382 \mathrm{pcm}$, respectively. Not only do the SANDY and NDaST results disagree on the uncertainty prediction, but the resulting $\mathrm{k}_{\mathrm{eff}}$ distribution is significantly non-Gaussian.

\subsubsection{Propagation of covariances for Legendre polynomial coefficients}

To further investigate the issue, SANDY was used to sample perturbations from:

1. a covariance matrix that includes all six Legendre polynomial coefficients (the default SANDY method); and

2. only the covariance matrix for P1 (to mimic NDaST) ${ }^{4}$.

These perturbed files were used to derive uncertainties and energy-correlations for the tabulated angular distribution of scattered neutrons for an incident-neutron energy of $1.8 \mathrm{MeV}$, where the fission neutron flux is the highest. This data is shown in Figure 3.

At this energy, the distribution is strongly peaked both forward and backward, a shape that requires a large number of polynomials to be accurately reproduced. As a consequence, the $\mathrm{k}_{\mathrm{eff}}$ sensitivity to $\mathrm{P} 1$ represents only a fraction of the total sensitivity to angular distributions, as it is described by a series of polynomial coefficients. Figure 3 shows that the contribution of $\mathrm{P} 1$ to the distribution uncertainty is only a small fraction of the collective uncertainty coming from all the terms, including their cross correlations.

The use of covariances over Legendre polynomials to represent uncertainties on angular distributions implicitly imposes strong correlations between the scattering angles. These reflect precisely the shape of the polynomials. Figure 3 shows that the use of only the P1 covariance matrix results in a full anti-correlation between all the forward and backward scattering angles. We confirmed in Figure 3 that the uncertainty and correlation matrix for the angular distribution are much different, both in uncertainties and correlations, when the full set of polynomial covariances are considered.

\subsection{2 $k_{\text {eff }}$ sensitivity to the Legendre polynomial coefficients}

The non-linearity of the PMF6 $\mathrm{k}_{\mathrm{eff}}$ with respect to variation of Legendre coefficients was investigated by sampling 1000 files with SANDY where only one Legendre polynomial coefficient was perturbed. To isolate the effects in each pertubation, a $10 \%$ standard deviation with full energy correlation was used for one coefficient in each study. The scatterplots of the results for P1 and P2 variation are shown in Figure 4, together with the $\mathrm{k}_{\text {eff }}$ distributions. From the quantile-quantile plot in Figure 5 one can see that the null-hypothesis of a Gaussian distribution is rejected, as confimed by the Shapiro-Wilks test.

The regression analysis of the scatterplots in Figure 4 shows that linearity is not an accurate approximation for these coefficients. The $\mathrm{k}_{\mathrm{eff}}$ results for $\mathrm{P} 2$ variation are even more notable in that an increase or decrease in the coefficient causes approximately the same increase in $\mathrm{k}_{\text {eff }}$. The use of a linear surrogate approach for $\mathrm{P} 2$ in this problem can give the false impression that $\mathrm{k}_{\mathrm{eff}}$ is not sensitive to the second Legendre polynomial coefficient, when the distribution is highly non-normal and the result of the unperturbed calculation is at one extreme of the distribution! Similar results were produced for all higher order coefficients that are present in the JEFF-3.3 library.

\footnotetext{
${ }^{4}$ The results obtained from this sampling are coherent with what NDaST predicted.
} 

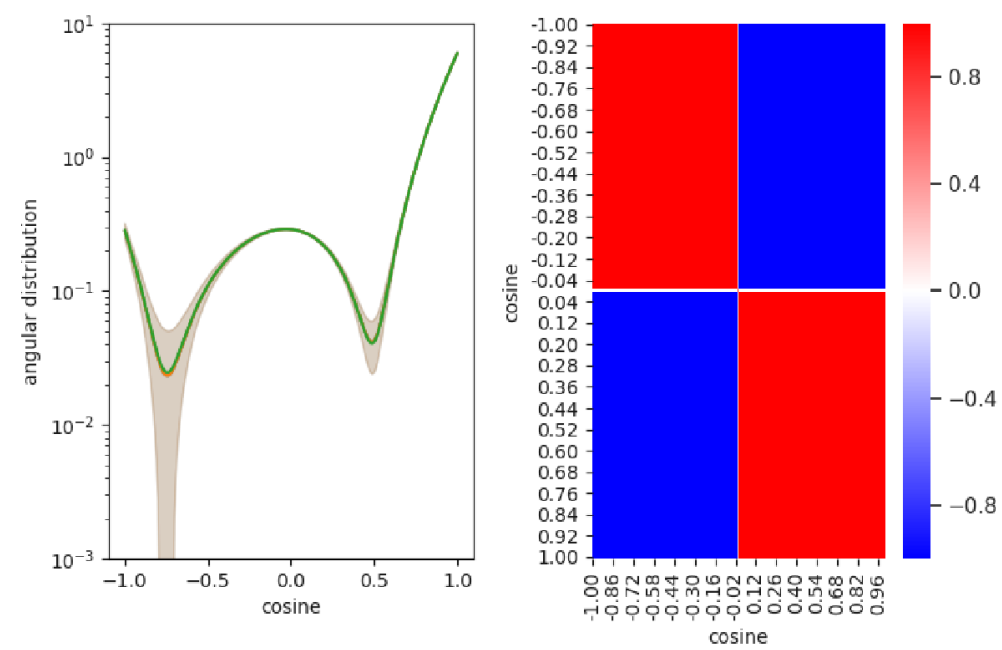

(a) Covariances were propagated for P1 only.
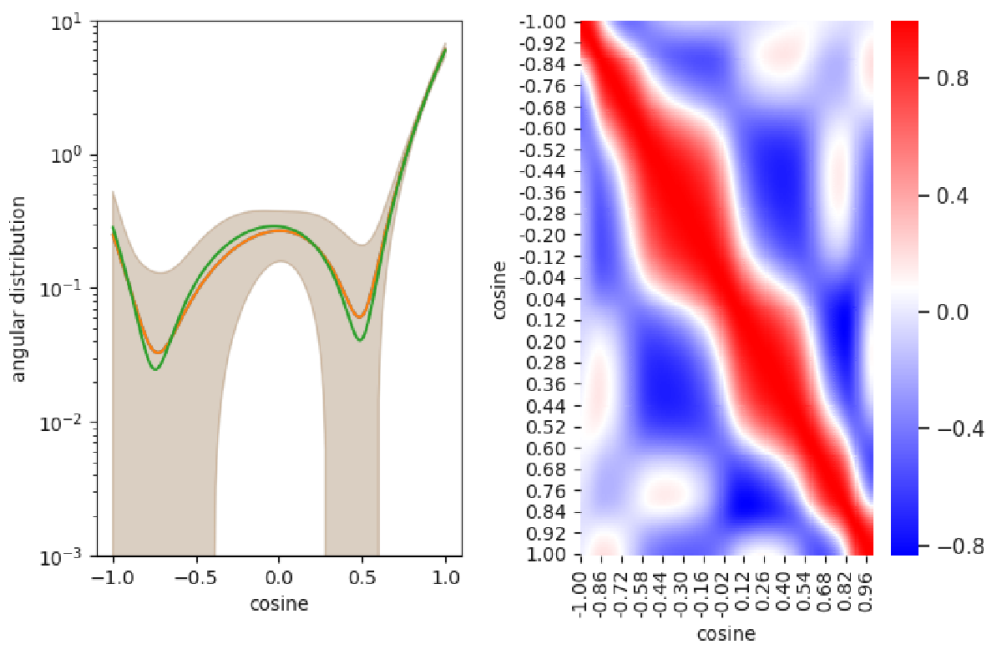

(b) Covariances were propagated for all Legendre polynomial coefficients.

Figure 3: ${ }^{238} \mathrm{U}$ elastic scattering angular distibution uncertainties and correlation matrices for incident-neutron energy $\mathrm{E}_{\mathrm{in}}=1.8 \mathrm{MeV}$. On the left, the green line represents the best estimate distribution, as derived from the evaluated file. The orange line is the average of the sampling, while the grey band is the associated standard deviation.

\section{Conclusions}

The JEFF-3.3 nuclear data library was processed with NJOY and SANDY to produce a suite of derived files for nuclear data uncertainty propagation. Uncertainties and other moments were quantified for a selection of ICSBEP cases using the NEA sensitivity tool NDaST and the Monte Carlo sampling code SANDY in combination with MCNP. The results show an excellent agreement between the two methodologies for the propagation of cross sections 

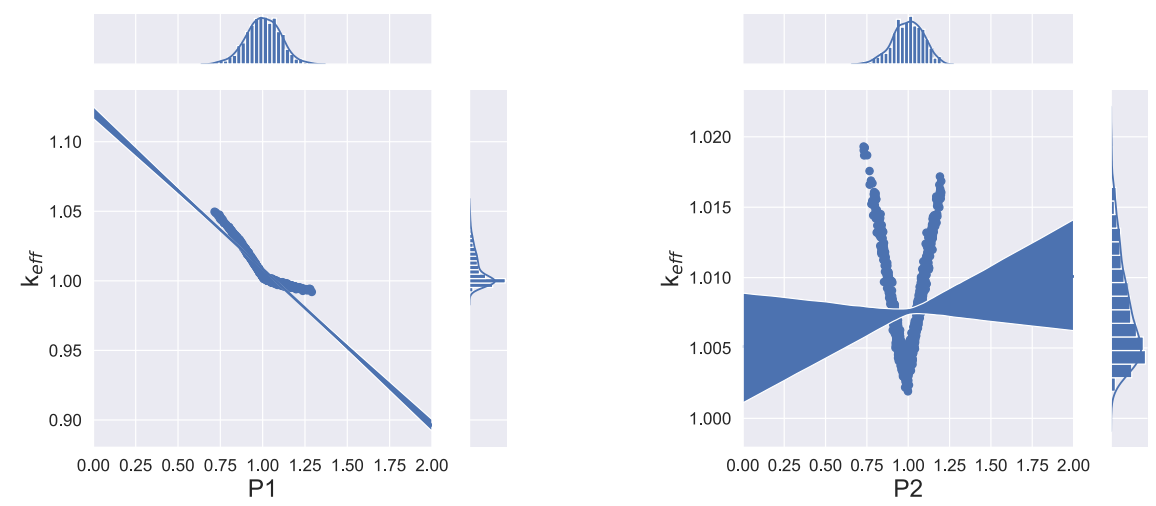

Figure 4: Estimates of the PMF6 $\mathrm{k}_{\mathrm{eff}}$ sensitivity to the P1 and P2 coefficients for elastic scattering in ${ }^{238} \mathrm{U}$.

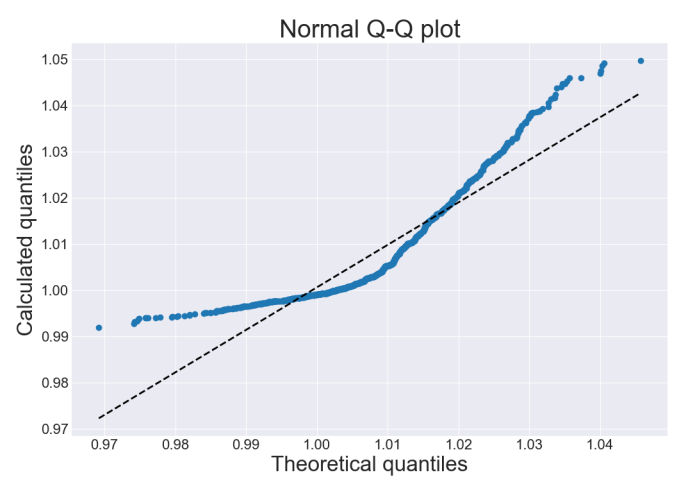

Figure 5: Quantile-quantile plot (Q-Q plot) of $\mathrm{k}_{\mathrm{eff}}$ produced with 300 perturbed $\mathrm{P} 1$ coefficients. The dashed line represents the Q-Q plot for a Normal PDF.

and fission neutron multiplicities covariances. Due to the limitations of the NJOY processing of prompt fission neutron spectra covariances that NDaST relies upon, differences were found with some benchmarks due to the propagation of this uncertainty.

Also, it was proved that the $\mathrm{k}_{\text {eff }}$ of strongly reflected systems is highly sensitive to the neutron angular distribution in the reflective material, and in particular to Legendre polynomial coefficients above the first order. The propagation of only P1 covariances largely underestimates the total effect of the elastic scattering angular distribution and introduces strong correlations between forward and backward scattering angles that are an artifact of the format and not based on experiment. In addition, the linearity assumption cannot be accurately employed for angular distribution in these and many other systems.

\section{References}

[1] J. Briggs, J. Bess, J. Gulliford, Nuclear Data Sheets 118, 396 (2014)

[2] C. Werner, Tech. Rep. LA-UR-17-29981 (2017) 
[3] B.T. Rearden, D.A. Reed, R.A. Lefebvre, D. Mueller, W.B.J. Marshall (2011)

[4] J.M. Ruggieri et al., ERANOS 2.1: International Code System for GEN IV Fast Reactor Analysis, in Proceedings of ICAPP 06 (2006), pp. 2432-2439

[5] J. Leppänen, M. Pusa, T. Viitanen, V. Valtavirta, T. Kaltiaisenaho, Annals of Nuclear Energy 82, 142 (2015), joint International Conference on Supercomputing in Nuclear Applications and Monte Carlo 2013, SNA + MC 2013. Pluri- and Trans-disciplinarity, Towards New Modeling and Numerical Simulation Paradigms

[6] L. Fiorito, G. Žerovnik, A. Stankovskiy, G.V. den Eynde, P. Labeau, Annals of Nuclear Energy 101, 359 (2017)

[7] M. Williams, G. Ilas, M. A. Jessee, B. Rearden, D. Wiarda, W. Zwermann, L. Gallner, M. Klein, B. Krzykacz-Hausmann, A. Pautz, Nuclear technology 183, 515 (2013)

[8] W. ZWERMANN, A. AURES, L. GALLNER, V. HANNSTEIN, B. KRZYKACZHAUSMANN, K. VELKOV, J. MARTINEZ, Nuclear Engineering and Technology 46, 343 (2014)

[9] O. Buss, A. Hoefer, J. Christian Neuber (2011), international Conference on Nuclear Criticality Safety (ICNC 2011)

[10] T. Zhu, A. Vasiliev, H. Ferroukhi, A. Pautz, S. Tarantola, Journal of Nuclear Science and Technology 52, 1000 (2015)

[11] A. Koning, D. Rochman, Nuclear Data Sheets 113, 2841 (2012), special Issue on Nuclear Reaction Data

[12] J. Dyrda, I. Hill, L. Fiorito, O. Cabellos, N. Soppera, EPJ Nuclear Sci. Technol. 4, 14 (2018)

[13] N. Soppera, M. Bossant, E. Dupont, Nuclear Data Sheets 120, 294 (2014)

[14] I. Hill, J. Gulliford, N. Soppera, M. Bossant, Dice 2013 new capabilities and data (2015)

[15] M. Herman, Members of the Cross Sections Evaluation Working Group (2009) 